\title{
MANUAL PARA PREVENIR LA DEPRESIÓN EN LOS ESTUDIANTES DE LA UNIVERSIDAD DEL ADULTO MAYOR
}

AUTORES: Caridad Isora Blanco Figueredo ${ }^{1}$

María Elena Sol Victortes ${ }^{2}$

Mario Zamora Pérez ${ }^{3}$

DIRECCIÓN PARA CORRESPONDENCIA: (iblancof@udg.co.cu)

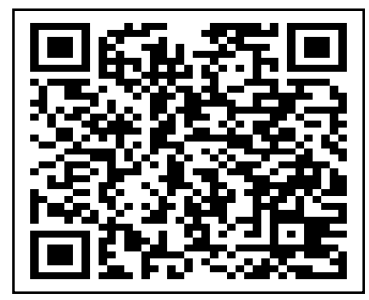

Fecha de recepción: 12/02/2021

Fecha de aceptación: 28/08/2021

\section{RESUMEN}

El presente manual consiste en una intervención grupal utilizada para prevenir la depresión y desmotivación en los estudiantes del adulto mayor del CUM Niquero. Teniendo en cuenta los beneficios que proporciona el trabajo con ellos, los profesores que atendemos a este grupo, nos apoyamos en el manual cognitivo conductual para fomentar un espacio de seguridad y confianza que propicie la actividad grupal en los mismos. El manual cuenta con ocho sesiones de trabajo, desglosada de la siguiente forma: una inicial, seis sesiones temáticas y una sesión final, en cada una de ellas se especifican actividades dirigidas al pensamiento como proceso cognitivo y los estados de ánimo en dichos estudiantes.

PALABRAS CLAVE: depresión; desmotivación; manual cognitivo conductual; prevenir.

\section{MANUAL TO PREVENT DEPRESSION IN STUDENTS OF THE UNIVERSITY OF THE ELDERLY}

\section{ABSTRACT}

This manual consists of a group intervention used to prevent depression and demotivation in the students of the older adult of CUM Niquero. Taking into account the benefits provided by working with them, the teachers that serve this group, we rely on the cognitive behavioral manual to promote a space of security and confidence that encourages group activity in them. The manual has eight work sessions, broken down as follows: an initial, six thematic sessions and a final session, each of which specifies activities aimed at thinking as cognitive process and moods in said students.

KEYWORDS: depression; demotivation; manual cognitive behavior; prevent.

\footnotetext{
1. Máster en la Educación Especial Profesora de Psicología. Centro Universitario Municipal Niquero. Universidad de Granma, Cuba. E-mail: iblancof@udg.co.cu

2 Máster en la Educación Técnico y Profesional. Profesora Coordinadora de la carrera de Agronomía. Centro Universitario Municipal Niquero. Universidad de Granma, Cuba. E-mail: msolv@udg.co.cu

${ }^{3}$ Máster en Ciencias Agrícolas. Profesor Coordinador de la carrera de Agronomía. Centro Universitario Municipal Campechuela. Universidad de Granma, Cuba. E-mail: mzamorap@udg.co.cu
}

(C) Universidad Estatal del Sur de Manabí. Jipijapa, Ecuador. 
Caridad Isora Blanco Figueredo, María Elena Sol Victortes, Mario Zamora Pérez

\section{INTRODUCCIÓN}

Uno de los objetivos fundamentales del proceso revolucionario cubano es ofrecer una mejor atención al Adulto Mayor, para la eliminación de enfermedades y prolongar su vida. Son muchos los factores que inciden en el desarrollo exitoso de las actividades que realizan los abuelos, entre ellas, la motivación juega un gran papel, al ser una de las condiciones para mantener la calidad de vida en estas personas, y aspirar a extender la existencia a 120 años, por lo que se requiere que las actividades se desarrollen de forma motivacional, ya que al presentar actividades que no estén acorde a sus necesidades, el Adulto Mayor se desvincula de estas, perdiendo la voluntad y el interés de asistir.

Como parte constante del perfeccionamiento del proceso de promoción de salud del Adulto Mayor, dirigido a que el practicante tenga cada vez una participación más activa como un agente transformador de su propio aprendizaje, en la actualidad los especialistas de esta materia se empeñan en la búsqueda de las vías, procedimientos y formas de enseñanza que garanticen un proceso que le permita al sujeto de esta edad, disfrutar de actividades amenas, creativas, acorde con sus intereses, al tiempo que las prepare para enfrentar esta etapa de la vida.

La Cátedra del Adulto Mayor de la Universidad de Granma en el municipio Niquero, fue constituida el 19 de noviembre de 2003, con una matrícula de 34 estudiantes. Representada por diferentes instituciones y organismos como la CTC, APC, Salud Pública, Cultura, INDER entre otras.

A partir de la aplicación del primer módulo de actividades docente educativas en el aula de la Cátedra Universitaria del Adulto Mayor en el año 2015-2016 del municipio Niquero, observamos que la mayoría de los estudiantes se desmotivaban y deprimían con facilidad. Presentaban estados de ánimo displacenteros fundamentalmente cuando en el hogar existían problemas con la familia y enfrentaban la muerte de algún ser querido.

Teniendo en cuenta los beneficios que proporciona el trabajo grupal, los profesores que atendemos al grupo, nos apoyamos en el manual cognitivo conductual el objetivo de: prevenir la depresión y fomentar un espacio de seguridad y confianza que propicie la actividad grupal en los estudiantes de la CUAM del municipio Niquero.

\section{DESARROLLO}

\section{Como nuestros pensamientos afectan nuestro estado de ánimo.}

\section{Sesión inicial}

1-Presentación. Nos presentaremos haciendo una rueda de nombres. Esto es, yo digo mi nombre, la persona a mi lado dice mi nombre y el suyo. La próxima persona dice mi nombre, el de la persona a mi lado y el suyo, y así sucesivamente.

2- Haremos la dinámica de la fiesta. El/LA facilitadora dice que va a dar una fiesta y que la persona que interese asistir debe traer algo consigo. La persona tiene que decir su nombre y lo que llevará a la fiesta. La clave es que, para ir a la fiesta, la persona debe llevar consigo algo que comience con la letra de su nombre. Al acertar la clave se le pide a la persona que comparta

26 UNESUM-Ciencias. Publicación cuatrimestral. Vol. 5, Año 2021, No. 5 (Septiembre-Diciembre) 
Publicación cuatrimestral. Vol. 5, No. 5 (Septiembre-Diciembre), Año 2021. Pág. 25-42 MANUAL PARA PREVENIR LA DEPRESIÓN EN LOS ESTUDIANTES DEL ADULTO MAYOR

algunos aspectos de ella para tomarlos en consideración a la hora de planificar la fiesta. Luego de algunos minutos de dinámica, y de algún modelaje por parte de los facilitadores, se procede a decir la clave para que aquellos que no la acertaron puedan "asistir a la fiesta".

3. El propósito de esta actividad es tratar la depresión a través de la enseñanza de formas diferentes para controlar mejor nuestro estado de ánimo. Existen cuatro metas:

- Disminuir o eliminar nuestros sentimientos de depresión

- Acortar el tiempo que estamos deprimidos

- Aprender formas de prevenir o evitar el deprimirse de nuevo

- Sentirnos más en control de nuestras vidas

4. La sesión de hoy tiene los siguientes propósitos:

- Ir conociéndonos mejor

- Aprender una forma útil de pensar acerca de la depresión

- Aprender cómo los pensamientos influyen en cómo nos sentimos

5. Una forma útil de pensar acerca de la depresión. (Fíjate en la hoja titulada como Entendemos la depresión) (Véase Apéndice A).

Los pensamientos afectan nuestro estado de ánimo:

El pensar ciertos tipos de pensamientos hace más o menos probable que te deprimas. Por "pensamientos" nosotros/as nos referimos a "cosas que nos decimos a nosotros/as mismos/as." (El/La facilitadora debe proporcionar un buen ejemplo)

- Los pensamientos pueden tener un efecto sobre tu cuerpo.

- Los pensamientos pueden tener un efecto sobre tus acciones.

- Los pensamientos pueden tener un efecto sobre tu estado de ánimo.

9. Una buena forma de pensar sobre este tipo de actividad es que aprenderás algunos métodos específicos para cambiar tus pensamientos y tus acciones, de tal forma que tu estado de ánimo mejorará.

\section{Tarea:}

1. El Termómetro del Animo (Apéndice B).

(Sesión 2). Como nuestros pensamientos afectan nuestro estado de ánimo.

Revisión de la tarea: termómetro del Ánimo: ¿Cómo te sentiste llenándolo cada día? ¿Tuviste alguna sorpresa? ¿Por qué es importante hacer los ejercicios?

1. Los pensamientos afectan nuestro estado de ánimo: 
Diferentes tipos de pensamientos producen diferentes efectos en tu estado de ánimo. Algunos pensamientos aumentan la posibilidad de que te deprimas, mientras que otros/as disminuyen dicha posibilidad.

2. ¿Qué son los pensamientos? (El/la facilitador hará esta pregunta de forma abierta facilitando una breve discusión al respecto.)

- Pensamientos son ideas (frases u oraciones) que nos decimos a nosotros/as mismos/as. Nosotros/as estamos todo el tiempo hablándonos mentalmente, pero no todo el tiempo nos damos cuenta de ello. Es útil pensar acerca de los pensamientos como "objetos" (ideas) que tienen un efecto real sobre nuestras mentes y cuerpos.

3. ¿Cómo suelen pensar las personas con depresión? (El/la facilitador hará esta pregunta de forma abierta, propiciando una lluvia de ideas en torno a pensamientos típicos de las personas con depresión. Algunos de los pensamientos que se generen como ejemplos serán utilizados posteriormente, clasificando los mismos de acuerdo al material que se presenta.).

Inflexible. Por ejemplo, una persona que está deprimida podrá pensar: “Tengo miedo”. "A mí siempre es al único que mandan a hacer las cosas en casa”. Un pensamiento flexible que podría evitar el sentirnos deprimidos/as podrá ser: “A veces tengo miedo, pero también a veces soy valiente". "Casi siempre me rechazan “.

Enjuiciador. Una persona que está deprimida podría pensar: "Soy un cobarde”, "soy feo” o "la gente no me quiere, no valgo" Un/a pensador/a flexible podría decir: "Soy más miedoso/a que la mayoría de la gente que yo conozco". "No seré el/la más lindo/a del mundo, pero tampoco soy, el/la más feo/a. Tengo otras cualidades que me hacen una persona agradable" o "no soy una monedita de oro para caerle bien a todo el mundo”.

4. ¿Cómo piensan los que no están deprimidos/as?

Cambian

Deprimido/a: "Yo siempre he sido y seguiré siendo un/a cobarde". "Soy y seguiré siendo un bruto". Flexible: "Me siento con miedo a veces, en algunas situaciones". "Si logro dar independiente podría sentirme mejor".

- Miran lo que hacemos, no lo que somos

El/la pensador/a deprimido/a podría decir: "Nací para sentirme mal” o "todo lo que hago me sale mal" o "tengo mala suerte". El/la pensador/a flexible podría decir "He estado comportándome de tal manera que ahora me siento deprimido/a”. "Todo el mundo tiene buenos y malos momentos”. "No tuve todo el apoyo o no puse todo el empeño para lograr lo que quería”.

- Tienen esperanzas para el cambio

El/la pensador/a deprimido/a diría: "Nunca me ha ayudado nada”. "Nada va a cambiar”. El/la pensador/a flexible diría: "Nada de lo que he tratado hasta ahora me ha ayudado, pero esto es nuevo y podría ser el tiempo oportuno para empezar a sentirme mejor". "Podría comenzar cambiando algunas cosas que están bajo mi control". "Voy a continuar tratando hasta que encuentre una solución”.

Tarea:

28 UNESUM-Ciencias. Publicación cuatrimestral. Vol. 5, Año 2021, No. 5 (Septiembre-Diciembre) 
Publicación cuatrimestral. Vol. 5, No. 5 (Septiembre-Diciembre), Año 2021. Pág. 25-42 MANUAL PARA PREVENIR LA DEPRESIÓN EN LOS ESTUDIANTES DEL ADULTO MAYOR

1. El Termómetro del ánimo.

2. Llena tu Lista de Pensamientos cada día.

\section{(Sesión 3). Usando tus pensamientos para cambiar tu estado de animo}

\section{Revisión de la tarea:}

A-Aumentando pensamientos que producen un mejor estado de ánimo.

Sugerencias para aumentar los pensamientos que permiten sentirnos mejor. Los siguientes puntos (sección A y B) serán presentados de tal manera que se genere una discusión grupal en torno a los mismos. Se proporcionará copia de ese material a los miembros del grupo.

1-Aumenta en tu mente el número de pensamientos buenos.

Haz una lista de los pensamientos buenos que tienes acerca de ti mismo/a y de la vida en general.

\section{Felicítate mentalmente.}

Las otras personas no notan la mayoría de las cosas que hacemos. Por lo tanto, es importante para nosotros/as reconocerlas y darnos crédito por ellas.

3. Detén todo lo que estás haciendo.

Cuando nos ponemos nerviosos/as podemos tomar un descanso y darnos mentalmente un "tiempecito" a nosotros/as mismos/as. ¡Tómate una pausa! Permite relajar tu mente. Permítele a tu mente tomar un respiro. Ponle atención a la habilidad natural de tu cuerpo de sentirse en paz. Sentirse en paz puede dar energías.

4- Proyección al futuro: Imagínate a ti mismo/a en el futuro, en un tiempo en el que las cosas serán mejores.

B-disminuyendo los pensamientos que nos hacen sentir mal.

1. Interrumpe tus pensamientos.

Cuando un pensamiento está afectando nuestro estado de ánimo, nosotros/as podemos identificarlo y tratar de interrumpirlo. Primero, identifica el pensamiento. Después, dile a ti mismo/a: "Este pensamiento está afectando mi estado de ánimo, por lo tanto, voy a pensar otro".

2. Tiempo para preocuparse.

Programa un "tiempo para preocuparte" cada día, de tal forma que te puedas concentrar enteramente en pensamientos necesarios y dejar el resto del día libre de preocupaciones. El “tiempo para preocuparte” puede ser de 10 a 30 minutos de duración diariamente.

3. Ríete de los problemas exagerándolos

Si tienes un buen sentido del humor, trata de reírte de tus preocupaciones. Si sientes que no tienes un buen sentido del humor, trata de hacerlo de cualquier forma. Algunas veces esto le puede quitar lo doloroso a algunas preocupaciones.

4. Considera lo peor que puede pasar 
Caridad Isora Blanco Figueredo, María Elena Sol Victortes, Mario Zamora Pérez

A menudo sucede que algunos miedos que tenemos acerca de lo que podría pasar nos hacen sentir más deprimidos/as que el miedo mismo que nos causa el analizar las cosas que nos atemorizan y enfrentar las peores posibilidades o consecuencias. Recuerda que lo peor que puede pasar es solamente una de las muchas posibilidades y sólo porque es la peor no significa que es la más probable que ocurra.

C. Siendo nuestro/a propio/a entrenador/a.

Así como nosotros/as podemos ayudar a alguien a hacer algo difícil dándoles instrucciones, también nosotros/as podemos ayudarnos a nosotros/as mismos/as al darnos instrucciones. Esto es lo que queremos decir cuando hablamos de aprender a sentirnos mejor.

Tiempo de práctica: Ahora pensemos en algunos ejemplos de cómo usar estas ideas. Piensa en cómo usar los ejemplos con los pensamientos que has tenido en esta semana.

D. Hablándole a tus pensamientos.

5. El método A-B-C-D (Apéndice E).

Cuando te sientas deprimido/a, pregúntate a ti mismo/a lo que estás pensando. Entonces trata de hablarle al pensamiento que te está molestando o hiriendo. Los siguientes ejemplos son presentados en transparencia y discutidos en el grupo. Luego se intentará generar un ejemplo grupal.

A es el evento Activante: es decir, lo que sucedió.

B es en inglés el "Belief” (la creencia) o el pensamiento que estás teniendo; es decir, lo que te dices a ti mismo/a acerca de lo que está pasando.

C es la Consecuencia de tu pensamiento; es decir, el sentimiento que tienes como resultado de tu pensamiento.

D es la forma en que le Discutes o le hablas a tu pensamiento

Tarea:

1. ¿Cómo me siento cada día? Continúa llenando el Termómetro del Ánimo.

2. Continúa trabajando en tus pensamientos. Llena tu Lista de Pensamientos cada día.

3. Usa el método A-B-C-D (Apéndice E) para hablarle a tus pensamientos.

(Sesión 4) Como tus pensamientos afectan tu estado de ánimo

Revisión de la tarea:

A. Algunos pensamientos que comúnmente pueden hacernos sentir deprimidos/as.

1. “Todos me deben querer."

2. Yo debo ser capaz de hacer las cosas bien siempre.

3. "Algunas personas son malas y deben ser castigadas."

4. "Me sentiré muy mal si las cosas no suceden en la forma en que deben suceder."

5. "Otras personas y cosas sobre las que no tengo control son las que me hacen sentir infeliz.”

30 UNESUM-Ciencias. Publicación cuatrimestral. Vol. 5, Año 2021, No. 5 (Septiembre-Diciembre) 
Publicación cuatrimestral. Vol. 5, No. 5 (Septiembre-Diciembre), Año 2021. Pág. 25-42 MANUAL PARA PREVENIR LA DEPRESIÓN EN LOS ESTUDIANTES DEL ADULTO MAYOR

Tarea:

1. ¿Cómo me siento cada día? Continúa llenando el Termómetro del Ánimo.

2. Continúa trabajando en tus pensamientos. Llena tu Lista de Pensamientos cada día.

3. Usa el método A-B-C-D para hablarle a tus pensamientos.

(Sesión 5). Como nuestras actividades afectan nuestro estado de ánimo

Revisión de la tarea:

1. Las actividades que realizamos afectan nuestro estado de ánimo: a través de nuestras actividades podemos evaluar nuestro estado de ánimo

Entre menos actividades agradables tiene la gente, más deprimidos/as se sienten.

La pregunta clave es: ¿Dejas de hacer cosas porque te sientes deprimido/a? o ¿Te deprimes porque dejas de hacer cosas? Estas preguntas se harán de forma abierta, generando una discusión grupal. La respuesta más probable es que sean las DOS cosas: Entre menos cosas agradables haces, más deprimido/a te sientes. Entre más deprimido/a te sientes, menos cosas agradables haces. A esto se le llama un "Círculo vicioso".

Para romper el círculo vicioso, puedes aumentar aquellas actividades que te hagan sentir mejor. A estas actividades también les puedes llamar "agradables", "placenteras", “reforzantes", “inspiradoras”, etc. Nosotros/as les llamamos “agradables”.

2. Las actividades agradables no tienen que ser actividades especiales (aunque pueden serlo). Por actividades agradables nosotros/as nos referimos principalmente a actividades cotidianas.

Algunas veces nos es difícil pensar en cosas que nosotros/consideramos agradables, especialmente si no las hemos hecho en mucho tiempo. Cuando estamos deprimidos/as, nos es más difícil recordar cosas agradables. Para ayudarte a hacerlo nosotros/as usamos la "Lista de actividades agradables” (Apéndice F).

- Algunos obstáculos para trabajar con actividades agradables:

1- Tus pensamientos:

2- La gente:

Ejercicio: Identifica actividades agradables que puedan ser añadidas a la lista. Si alguna de las actividades no se aplica a ti, entonces déjala en blanco o táchala con una línea. Es probable que nunca hayas hecho esto anteriormente en tu vida. Esta semana no tienes que hacer nada más allá de lo que ordinariamente haces. Nosotros/as queremos saber el número de actividades agradables que tienes cada día en el curso de la próxima semana.

Tarea

1. El Termómetro del Ánimo

2. El método ABCD

(Sesión 6). Como nuestras actividades afectan nuestro estado de ánimo. 
Revisión de la tarea:

1. ¿Cómo es que las actividades agradables te pueden ayudar a cambiar tu estado de ánimo?

Se volverá a presentar la transparencia con el diagrama que representa la interacción entre pensar, actuar y sentir. No es suficiente decirte a ti mismo/a: ¡Siéntete bien! A menudo es más fácil cambiar las cosas que haces. Si cambias las cosas que haces, entonces también cambiarás la forma en que te sientes.

2. ¿Qué son actividades agradables?

Actividades agradables no necesitan ser actividades especiales, aunque éstas pueden ser especiales. A menudo las actividades agradables son actividades habituales que nos hacen disfrutar. Ejemplos de éstas pueden ser: observar una puesta del sol, leer un libro, hablar con un amigo/a ya sea en persona o por teléfono, ir a un parque, oler una flor, escuchar música, dibujar, etcétera. Las actividades agradables pueden ser diferentes para diferentes personas. Por ejemplo, algunas personas encuentran que leer un libro a solas es una actividad muy agradable. Otras personas pueden encontrar divertido el estar en un centro con mucho ruido y con mucha gente.

3. Para sentirnos saludables emocionalmente es importante tener un balance adecuado de

Actividades agradables. Nuestro estado de ánimo está en lo óptimo cuando nuestras actividades están bien balanceadas entre las cosas que "tenemos que hacer" y las cosa que "queremos hacer". Puesto que nosotros/as tenemos más poder de elección sobre las cosas que queremos hacer, es importante tener estas actividades en mente y hacerlas.

Tarea:

1. El Termómetro del Ánimo.

2. Al finalizar cada día, ponle una marca a cada una de las actividades de la Lista de Actividades Agradables que tuviste. Para cada día anota el número total de marcas al final de la lista. Trae la lista contigo la próxima semana.

3. Un experimento contigo mismo/a.

Llenar la hoja titulada: ¿Cuánto crees que disfrutarás de tus actividades agradables? (Apéndice I) Lo que esperamos que aprendas de este experimento es que: (presentar los objetivos en transparencia)

a. No necesitas esperar hasta que sientas las energías de hacer algo para hacerlo.

b. Puedes escoger hacer algo y realmente hacerlo.

c. Puedes disfrutar algunas actividades aun cuando pensaste que no podrías hacerlo.

d. Puedes influir tu estado de ánimo con tus actividades.

(Entre más practiques esto, más cambios obtendrás en tu estado de ánimo)

(Sesión 7). Como nuestras actividades afectan nuestro estado de ánimo.

Revisión de la tarea:

Creando tu propio plan para superar la depresión:

32 UNESUM-Ciencias. Publicación cuatrimestral. Vol. 5, Año 2021, No. 5 (Septiembre-Diciembre) 
Publicación cuatrimestral. Vol. 5, No. 5 (Septiembre-Diciembre), Año 2021. Pág. 25-42 MANUAL PARA PREVENIR LA DEPRESIÓN EN LOS ESTUDIANTES DEL ADULTO MAYOR

1. ¿Qué son las metas? ¿Cuál es la relación de las metas con las actividades? El/la facilitadora hará estas preguntas de forma abierta, fomentando la discusión grupal.

2. Para superar la depresión:

-Ponte metas razonables

-Anota las cosas positivas que haces

-Recompénsate en tu mente y en la vida real

2. Poniéndote metas realistas: Ponte metas claras y concretas (de tal forma que te des cuenta cuando las hayas realizado)

3- Hay varios tipos de metas:

Metas a corto plazo. Cosas que te gustaría hacer pronto (digamos que dentro de los próximos seis meses)

Metas a largo plazo. Cosas que te gustaría hacer en alguna ocasión en tu vida.

Metas de la vida. Filosofía de la vida: ¿Qué es lo que te importa más en la vida?

¿Cuáles son tus metas? ¿Cuáles son los obstáculos que te impiden realizarlas? El/la facilitador repartirá papel y lápiz a cada miembro del grupo. Se le pedirá a cada uno/a que escriba una meta a corto plazo y una meta a largo plazo. Se le pedirá al grupo que voluntariamente compartan las metas que han escrito y que se analice si las mismas son realistas. Así mismo, que se identifiquen los posibles obstáculos que puedan impedir el realizarlas.

En el día de hoy sólo pensaremos en metas y planes individuales, es decir, metas y planes que sólo tienen que ver contigo. El/la facilitador repartirá papel y lápiz a cada miembro del grupo. Estos/as deberán visualizar un objetivo (meta) e identificar pasos para obtenerlo

Tarea:

1. El Termómetro del Ánimo.

2. Tu Programa Semanal de Actividades.

3. Manejo del tiempo: Haz una lista de las metas que te gustaría hacer esta semana. Ponlas en orden de prioridad. ¿Estás seguro/a de que realmente quieres hacer las últimas de tu lista? Identifica un plan para lograr las de mayor prioridad.

4. El método ABCD

(Sesión 8). Nuestras actividades afectan nuestro estado de ánimo.

Revisión de la tarea:

La depresión y el manejo saludable de la realidad.

1. Todos/as nosotros/as vivimos en dos mundos:

A. El mundo objetivo (el mundo de afuera, lo que está afuera de nosotros/as)

B. El mundo subjetivo (el mundo de adentro, lo que está adentro de nuestra mente) 
El/la facilitadora presentará en laminilla el "retrato de la dama” Se generará una discusión grupal en torno a diversas percepciones que se pueden tener del mismo dibujo (el retrato de una dama joven o el de una anciana). Todo esto dirigido a ilustrar la diferencia entre los mundos objetivo y subjetivo.

2. Estos dos mundos son nuestra REALIDAD. La clave para sentirte emocionalmente saludable es:

Aprender a manejar estas dos partes de nuestra realidad

3. Nadie controla completamente estos dos mundos. Sin embargo, podemos aprender a:

Obtener un mayor control del mundo subjetivo y a identificar formas de manejo del mundo objetivo. Cuando estamos deprimidos/as sentimos que no tenemos ningún control

4. Formas de sentir más control:

A. Alternativas: Entre más alternativas aprendes a tener, mayor libertad tendrás. El/la facilitadora le presentará la siguiere situación al grupo, pidiendo alternativas para la misma: Hay peña del danzón y te invitan.

B. Pensando que la vida está hecha de pequeños trozos de tiempo: También ten cuidado de decirte a ti mismo/a:

C. "Yo no puedo disfrutar de la vida hasta que ”.

Por ejemplo, si te dices: "Yo no puedo disfrutar de la vida hasta que esta depresión se termine" o "yo no voy a disfrutar del baile de danzón hasta tanto no pertenezca al grupo del adulto mayor"; podrías no hacer el tipo de cosas que te ayudarían a liberarte de la depresión más prontamente.”

Tiempo de despedida:

Se procederá a realizar una postal grupal en la que cada adulto mayor será libre de expresarse a sus demás compañeros/as. Se utilizarán varios materiales tales como cartulina, escarcha, goma de pegar, lápices de colores y crayolas.

Valoración de los resultados obtenidos: Las actividades desarrolladas con 9 abuelos en estado depresivo permitieron que 7 de ellos se rehabilitaran y en 2 abuelos se evidencian resultados favorables, se debe seguir aplicando actividades para lograr su restablecimiento total.

La aplicación de las actividades del manual demostró que:

1-Una buena forma de pensar, permite mejorar los estados de ánimo.

2-Las actividades cotidianas afectan en el adulto mayor su realización personal, influyendo de esta forma en su estado de ánimo.

3- El manejo saludable de la realidad es un factor determinante para combatir

\section{CONCLUSIONES}

Al sistematizar las actividades del manual elaborado para prevenir la depresión en los estudiantes del aula de la Cátedra Universitaria del Adulto Mayor de la Filial Universitaria Municipal Niquero, y la evaluación realizada se define las siguientes conclusiones:

34 UNESUM-Ciencias. Publicación cuatrimestral. Vol. 5, Año 2021, No. 5 (Septiembre-Diciembre) 
Publicación cuatrimestral. Vol. 5, No. 5 (Septiembre-Diciembre), Año 2021. Pág. 25-42 MANUAL PARA PREVENIR LA DEPRESIÓN EN LOS ESTUDIANTES DEL ADULTO MAYOR

La aplicación de las actividades debe realizarse en una secuencia lógica como lo indica la metodología del manual, lo que ha permitido resultados favorables en los abuelos deprimidos de la muestra seleccionada.

Mediante la implementación del manual se logró fomentar un espacio de seguridad y confianza para la actividad grupal en los estudiantes de la CUAM del municipio Niquero.

\section{REFERENCIAS BIBLIOGRÁFICAS}

Ávila Fuentes, Martha. (2002). El grupo y su estudio en Psicología Social. Editorial ENPES, La Habana, Cuba.

Lomov, B. F. (2002). El problema de la comunicación en Psicología. Editorial Ciencias Sociales, Ciudad de la Habana, Cuba.

Castellanos, C, R. (2003). Psicología. Selección de Textos. Editorial Félix Varela, Ciudad de la Habana, 2003.

Colectivo de autores: Dinámicas de Grupos en Educación. Editorial Pueblo y Educación, Ciudad de la Habana, 2002.

Colectivo de autores: Metodología de la investigación Social. . Editorial Félix Varela, Ciudad de la Habana, Cuba.

Colectivo de autores: (2001). Psicología para educadores. Editorial Pueblo y Educación, Ciudad de la Habana, Cuba.

Colectivo de autores: (2002). Selección de temas psicopedagógicos. Editorial Pueblo y Educación, Ciudad de la Habana, Cuba.

Cruz, L. (2002). Selección de Lecturas de Psicología del Desarrollo. Editorial Varona, Ciudad de la Habana, Cuba.

Núñez Aragón, Elsa y otros. (1994). Manual del Programa de Educación Comunitaria. Editorial Pueblo y Educación, Ciudad de la Habana, Cuba.

\section{Apéndice A}

\section{¿Cómo entendemos la depresión?}

\section{Pensar}

Sentir Actuar

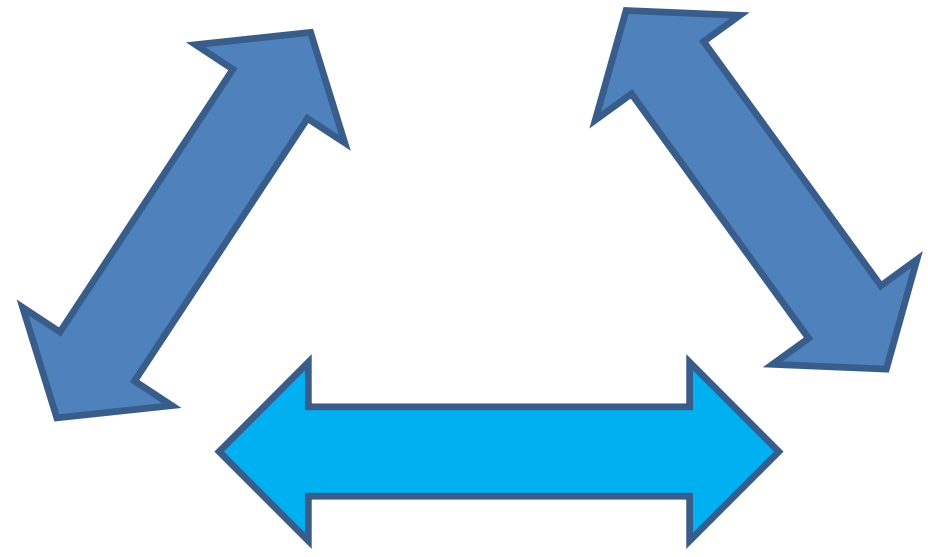

\section{Apéndice B}

\section{Termómetro del Ánimo}

\begin{tabular}{|l|c|c|c|l|c|c|c|}
\hline Ánimo & Domingo & Lunes & Martes & $\begin{array}{l}\text { Miércole } \\
\text { s }\end{array}$ & Jueves & Viernes & Sábado \\
\hline El Mejor & 9 & 9 & 9 & 9 & 9 & 9 & 9 \\
\hline
\end{tabular}


Caridad Isora Blanco Figueredo, María Elena Sol Victortes, Mario Zamora Pérez

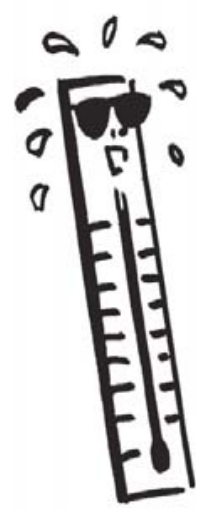

\begin{tabular}{|l|c|c|c|c|c|c|c|}
\hline Bastante Mejor & 8 & 8 & 8 & 8 & 8 & 8 & 8 \\
\hline Mejor & 7 & 7 & 7 & 7 & 7 & 7 & 7 \\
\hline Mejor que Regular & 6 & 6 & 6 & 6 & 6 & 6 & 6 \\
\hline Regular & 5 & 5 & 5 & 5 & 5 & 5 & 5 \\
\hline Peor que Regular & 4 & 4 & 4 & 4 & 4 & 4 & 4 \\
\hline Peor & 3 & 3 & 3 & 3 & 3 & 3 & 3 \\
\hline Bastante Peor & 2 & 2 & 2 & 2 & 2 & 2 & 2 \\
\hline El Peor & 1 & 1 & 1 & 1 & 1 & 1 & 1 \\
\hline
\end{tabular}

\section{Apéndice C}

\section{Lista de pensamientos negativos}

Haz una marca de cotejo (x) a los pensamientos negativos que tuviste cada día.

\begin{tabular}{|l|l|l|l|l|l|l|l|}
\hline & D & L & M & M & J & V & S \\
\hline Estoy confundido/a. & & & & & & & \\
\hline No existe amor en el mundo & & & & & & & \\
\hline Estoy echando a perder mi vida. & & & & & & & \\
\hline Tengo miedo. & & & & & & & \\
\hline Terminaré solo/a. & & & & & & & \\
\hline Nadie considera importante la amistad. & & & & & & & \\
\hline No tengo paciencia. & & & & & & & \\
\hline Nada vale la pena. & & & & & & & \\
\hline No tiene sentido seguir con esto. & & & & & & & \\
\hline Soy un/a estúpido/a & & & & & & \\
\hline $\begin{array}{l}\text { Cualquiera que piense que soy agradable es } \\
\text { porque no me conoce de verdad. }\end{array}$ & & & & & & & \\
\hline La vida no tiene sentido. & & & & & & & \\
\hline Soy feo/a. & & & & & & & \\
\hline No puedo expresar mis sentimientos. & & & & & \\
\hline Nunca encontraré lo que realmente quiero. & & & & & \\
\hline
\end{tabular}

36 UNESUM-Ciencias. Publicación cuatrimestral. Vol. 5, Año 2021, No. 5 (Septiembre-Diciembre) 
No soy capaz de querer.

No valgo nada.

Ya no tengo esperanzas.

No sirvo para nada.

No voy a poder resolver mis problemas.

Todo lo que hago me sale mal.

Nunca podré cambiar.

Todo se me daña.

Ya no me entusiasma nada.

Quisiera no haber nacido.

No voy a poder dormir.

Soy menos que otras personas.

Todo es mi culpa.

¿Por qué me pasan tantas cosas malas?

Nada me divierte.

La vida no vale la pena.

Nunca saldré de esta depresión.

No tengo suficiente fuerza de voluntad.

Quisiera estar muerto/a.

Me pregunto si la gente habla de mí.

Las cosas se van a poner cada vez peor.

Tengo mal carácter

La vida es injusta.

No me atrevo imaginarme lo que será mi vida dentro de 10 años.

Algo está mal en mí.

Soy muy egoísta.

No soy tan bueno/a como otros/as.

Me siento herido/a fácilmente.

Nadie en mi familia me entiende. 
Siempre estoy aburrido/a.

Total de Pensamientos Negativos

\section{Apéndice D}

\section{Lista de pensamientos positivos}

Haz una marca de cotejo (x) a los pensamientos positivos que tuviste cada día

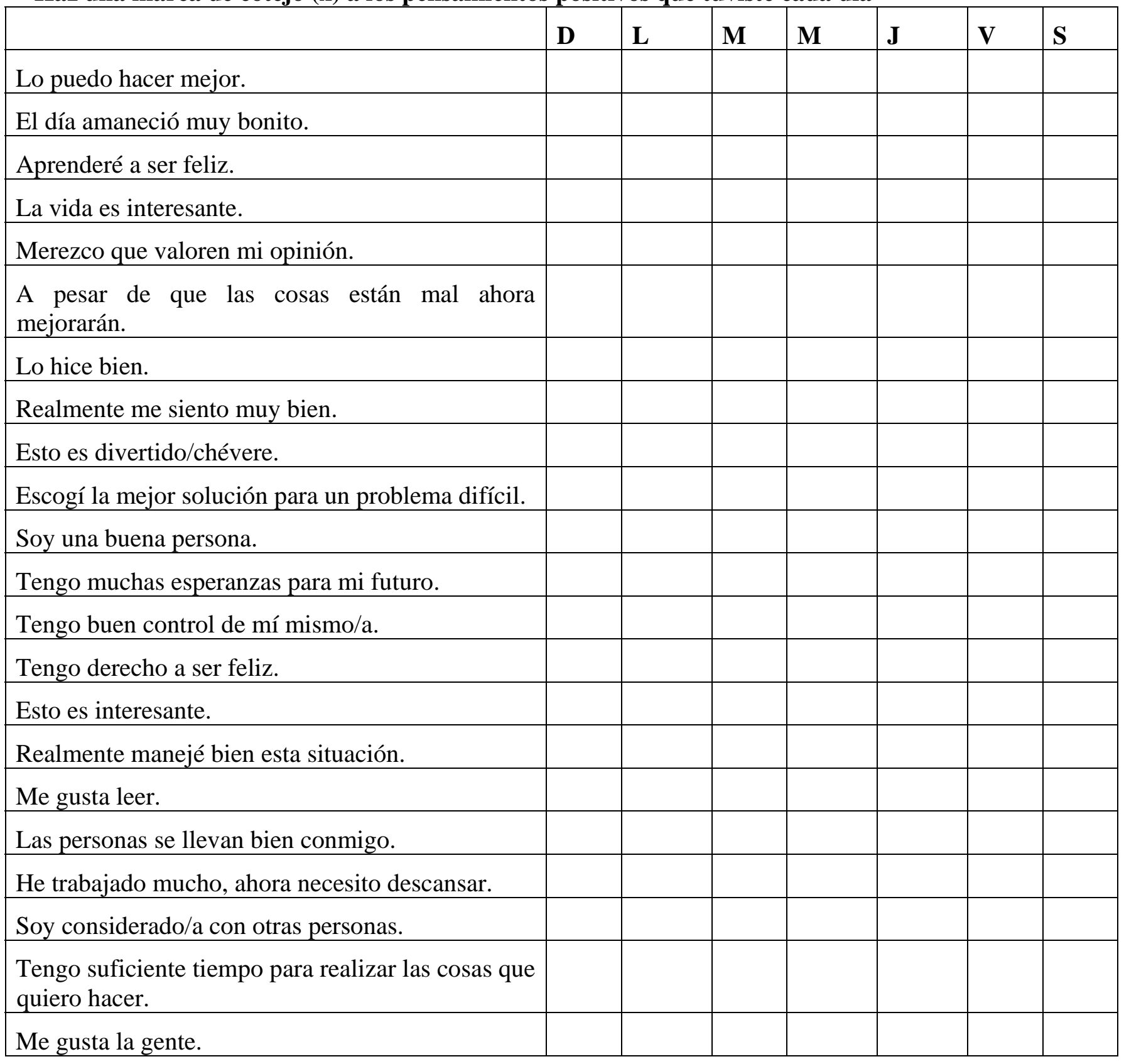

38 UNESUM-Ciencias. Publicación cuatrimestral. Vol. 5, Año 2021, No. 5 (Septiembre-Diciembre) 


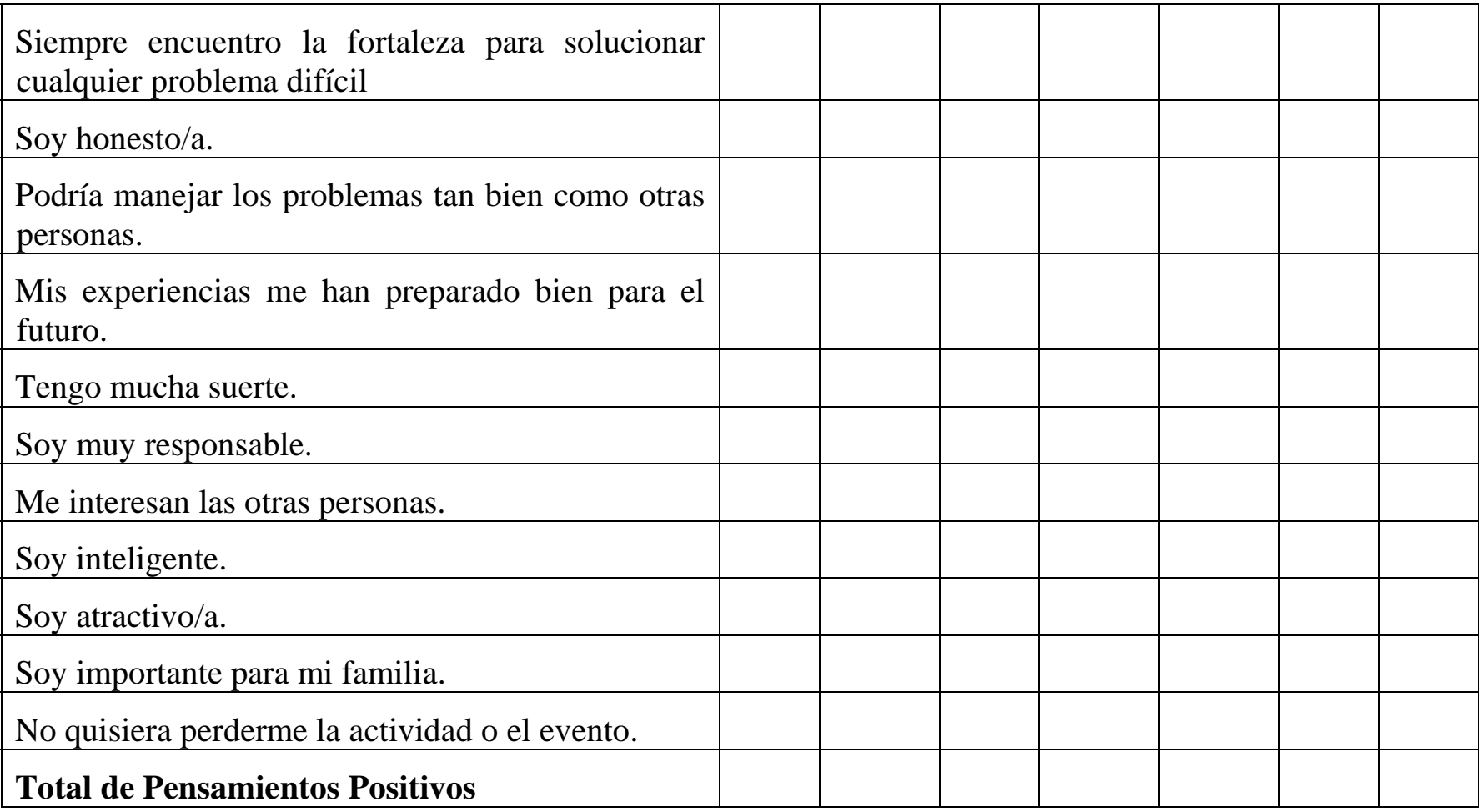

\section{Apéndice E}

\section{Trabajando con el método A B C D}

A. Evento Activante (¿Qué sucedió?)

C. Consecuencia (¿Cómo reaccionaste?) 
B. Belief (Creencia de lo que sucedió, lo que te dijiste acerca de lo que sucedió y lo que influyó tu reacción)
D. Discutir o Debatir la Creencia (Los pensamientos alternos que podrían ayudarte a reaccionar de una forma más saludable)

\section{Apéndice $\mathbf{F}$}

\section{Lista de actividades agradables}

Pon una marca (x) a las actividades agradables que realizaste cada día.

\begin{tabular}{|l|l|l|l|l|l|l|l|}
\hline & D & L & M & M & J & V & S \\
\hline Ponerme ropa que me gusta & & & & & & & \\
\hline Estar fuera de casa (playa, campo) & & & & & & & \\
\hline $\begin{array}{l}\text { Ayudar a grupos que respeto (ej. iglesia, } \\
\text { comunidad) }\end{array}$ & & & & & & & \\
\hline Hablar de deportes & & & & & & & \\
\hline Ver o participar en deportes & & & & & & & \\
\hline Ir al teatro, concierto, bailar & & & & & & & \\
\hline Planificar viajes o vacaciones & & & & & & & \\
\hline Comprar cosas & & & & & & & \\
\hline Hacer artesanías/manualidades & & & & & & & \\
\hline Asistir a la iglesia & & & & & & & \\
\hline Arreglar mi cuarto y mi casa & & & & & & & \\
\hline Leer libros, revistas o periódicos & & & & & & & \\
\hline Conversar & & & & & & & \\
\hline Jugar, billar & & & & & & & \\
\hline Ver televisión & & & & & & & \\
\hline Arreglar carros, bicicletas & & & & & & \\
\hline Terminar una tarea difícil & & & & & \\
\hline Montar un rompecabezas & & & & & \\
\hline Darme un baño caliente & & & & & \\
\hline Escribir historias, poesía, música, en un diario & & & & & \\
\hline
\end{tabular}

40 UNESUM-Ciencias. Publicación cuatrimestral. Vol. 5, Año 2021, No. 5 (Septiembre-Diciembre) 
Publicación cuatrimestral. Vol. 5, No. 5 (Septiembre-Diciembre), Año 2021. Pág. 25-42 MANUAL PARA PREVENIR LA DEPRESIÓN EN LOS ESTUDIANTES DEL ADULTO MAYOR

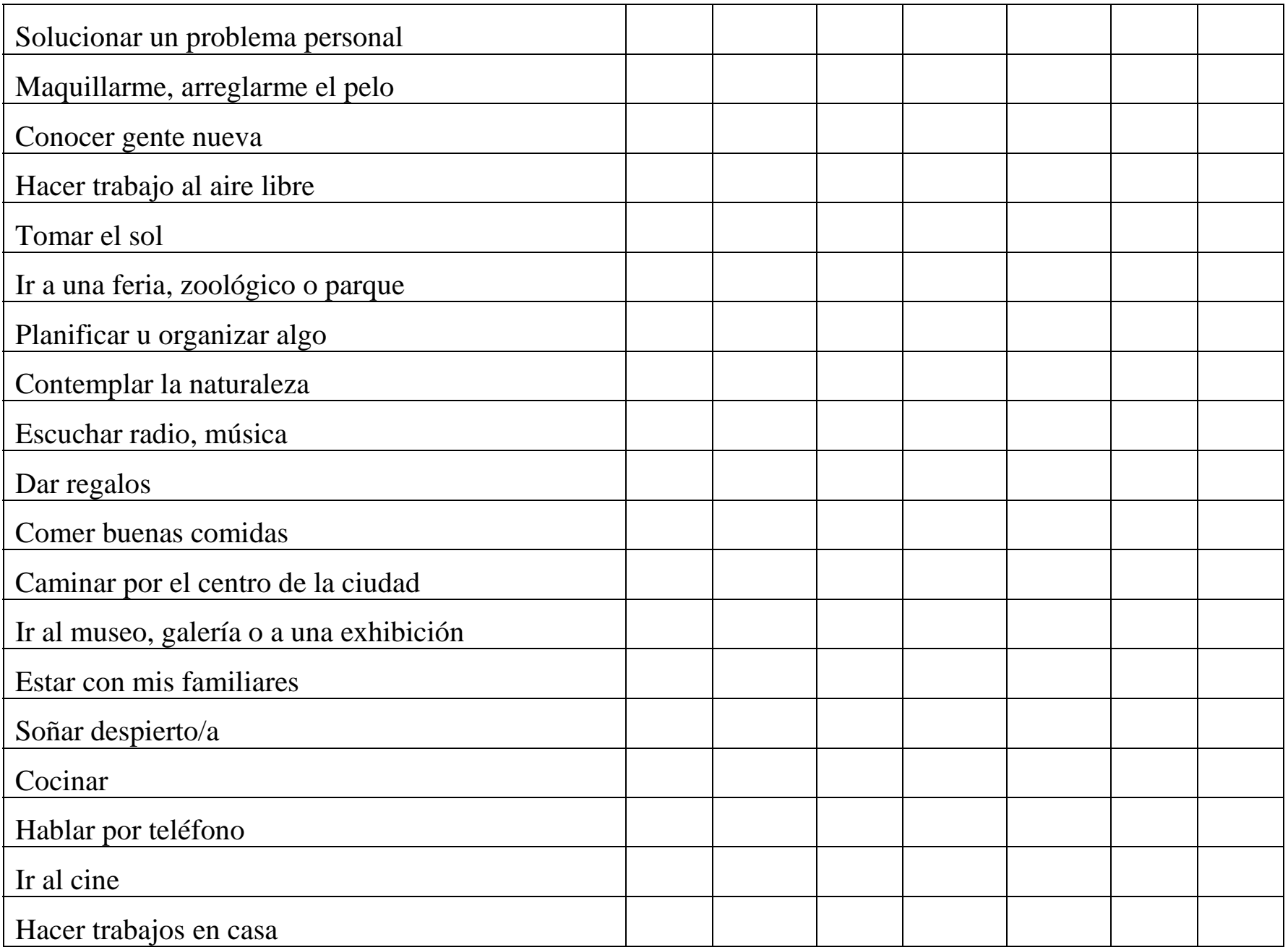


Caridad Isora Blanco Figueredo, María Elena Sol Victortes, Mario Zamora Pérez

42 UNESUM-Ciencias. Publicación cuatrimestral. Vol. 5, Año 2021, No. 5 (Septiembre-Diciembre) 